\title{
Fischöl bremst Wachstum von Prostatatumoren
}

\author{
Prostatatumoren reagieren offenbar auf die Zusammensetzung der \\ Ernährung: Unter einer fettarmen Diät mit einem Zusatz von ungesättigten \\ Omega-3-Fettsäuren teilen sie sich langsamer als unter der üblichen \\ westlichen Ernährung.
}

$\mathrm{M}$ it einer ungewöhnlichen Studie haben US-Forscher aus Los Angeles nachgewiesen, dass die Ernährung das Teilungsverhalten von Prostatatumorzellen beeinflusst. Ein Team um William Aronson hat 55 Männern mit Prostatakarzinom vier bis sechs Wochen vor der Entfernung der Drüse unterschiedliche Mahlzeiten aufgetischt: Etwa die Hälfte bekam die übliche US-amerikanische Diät, bei der $40 \%$ der Kalorien überwiegend aus gesättigten Fettsäuren stammten, das Verhältnis von Omega-6- zu Omega3-Fettsäuren betrug 15:1. Die andere Hälfte bekam eine fettreduzierte Diät, hier stammten nur $15 \%$ der Kalorien aus Fetten, und das Verhältnis von Omega-6- zu Omega-3-Fettsäuren lag bei 2:1. Um dieses günstige Verhältnis zu erreichen, erhielten die Teilnehmer in dieser Grup- pe zusätzlich täglich $5 \mathrm{~g}$ Fischöl. Die Mahlzeiten wurden von Klinikköchen zubereitet und den Männern zugestellt.

Nach der Prostatektomie nahmen die Forscher die Tumorzellen genauer unter die Lupe. Sie stellten fest, dass sich bei Männern mit der Fischöldiät in den Zellmembranen das Fettsäureverhältnis zugunsten von Omega-3-Fettsäuren verschoben hatte. Noch interessanter war jedoch das Teilungsverhalten der Zellen: Mit der Fischöldiät lag die Teilungsrate der Krebszellen ein Drittel unter der von Männern mit normaler Ernährung. Dies ist insofern relevant, als eine hohe Teilungsrate mit einer ungünstigen Prognose und einem erhöhten Metastasierungsrisiko einhergeht. Auch das Rezidivrisiko nach der Prostataoperation ist erhöht, wenn sich die Tumorzellen rasch teilen.

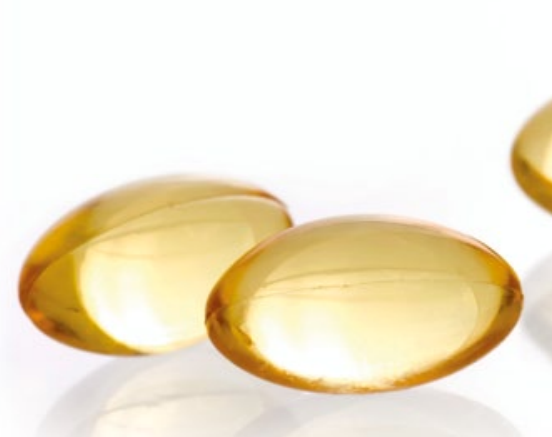

Verwendeten die Wissenschaftler nun Serum der Männer mit der Fischöldiät, so konnte dieses die Teilung von Krebszellen in vitro ebenfalls bremsen, nicht aber Serum der Männer mit der herkömmlichen Ernährung. Über welche Faktoren der günstige Effekt vermittelt wird, ist allerdings noch unklar. Die Forscher hatten zunächst gehofft, mit der Fischöldiät die Konzentrationen des proliferationsfördernden Wachstumsfaktors IGF-1 im Blut zu verringern, dies war aber nicht der Fall. Sie vermuten daher andere Mechanismen.

Thomas Müller

Aronson WJ et al. Phase II prospective randomized trial of a low-fat diet with fish oil supplementation in men undergoing radical prostatectomy. Cancer Prev Res (Phila). 2011;4(12):2062-71.

\section{Osteosarkom: viertes Zytostatikum bringt nichts}

Intensive primäre Chemotherapie erhöht zwar den Anteil der Osteosarkome, die histologisch gut ansprechen. Sie ist jedoch, wie zahlreiche Studiendaten zeigten, nicht unbedingt mit besseren Überlebenschancen verbunden.

S eit Einführung der Chemotherapie in die Behandlung des Osteosarkoms wurden keine substanziellen Verbesserungen beim Überleben mehr erzielt. In einer systematischen Literaturrecherche zur systemischen Therapie lokalisierter, hochgradiger Osteosarkome wurden randomisierte wie nichtrandomisierte Studien berücksichtigt. Zu Vergleichszwecken zog man historische klinische Studien der Prä-Chemotherapie-Ära heran. Die Langzeitüberlebensrate nach lokaler Tumortherapie allein war 16\% (9-23\%). Durch (neo)adjuvante Chemotherapie erreichte man eine längere Überlebenszeit; die Dauer bis zum Auftreten von Metastasen war 1,5- bis 2-mal so lang.

Um das Ansprechen auf einzelne Vertreter der häufig beim Osteosarkom ein- gesetzten Zytostatika vergleichen zu können, nahmen sich die Autoren nochmals die Phase-II-Studien vor, in denen Adriamycin (A), Methotrexat (M), Cisplatin (P), Ifosfamid (Ifo) und Etoposid (E) bei vorbehandelten, rezidivierten oder refraktären Tumoren in Monotherapie geprüft wurden. In sämtlichen Studien gab es hohe Ansprechraten von $26 \%(\mathrm{P}), 32 \%$ (M), $33 \%$ (Ifo) bzw. $43 \%$ (A). Lediglich auf E sprachen nur $4 \%$ der Sarkome an.

Unterschiedliche Zwei-, Drei- und Vierfach-Protokolle, wie sie von amerikanischen und europäischen Studiengruppen angewendet wurden, untersuchte man in einer Metaanalyse. In 19 Studien mit neoadjuvanter Therapie betrug die mediane 5-Jahres-Rate ereignisfreien Überlebens bei Einsatz von zwei Substan- zen $48 \%$ - gegenüber $58 \%$ unter Polychemotherapien mit drei oder mehr Zytostatika. Das 5-Jahres-Gesamtüberleben betrug 62 resp. $70 \%$.

Sowohl das ereignisfreie als auch das Gesamtüberleben wurden durch eine Dreifach-Therapie im Vergleich zu einem dualen Regime signifikant verlängert (Hazard Ratio 0,701 resp. 0,792). Die Behandlungserfolge unterschieden sich nicht signifikant, wenn statt einer Triple-Therapie ein Protokoll mit vier verschiedenen Substanzen eingesetzt wurde.

Fazit: Die Erweiterung des Protokolls aus den Wirkstoffen Methothrexat, Adriamycin und Cisplatin um eine vierte Substanz bietet demnach keinen Vorteil. Offen ist weiterhin, ob eine Dosisintensivierung nach einem schlechten Ansprechen auf präoperative Chemotherapie die Prognose verbessert. Waldtraud Paukstadt

Anninga JK et al. Chemotherapeutic adjuvant treatment for osteosarcoma: where do we stand? Eur J Cancer. 2011;47(16):2431-45. 